\title{
Prediction of prognosis in acute paraquat poisoning using severity scoring system in emergency department
}

\author{
YOUNG-GI MIN ${ }^{1}$, JUNG HWAN AHN ${ }^{1}$, YIU CHEUNG CHAN ${ }^{2}$, SZE HONG NG ${ }^{2}$, MAN LI TSE ${ }^{2}$, FEI LUNG LAU ${ }^{2}$, and \\ CHI KEUNG CHAN ${ }^{2}$ \\ ${ }^{1}$ Department of Emergency Medicine, Ajou University School of Medicine, San5, Wonchun-dong, Youngtong-gu, Suwon, \\ Republic of Korea \\ ${ }^{2}$ Hong Kong Poison Information Center, United Christian Hospital, 130 Hip Wo Street, Kwun Tong, Kowloon, Hong Kong
}

\begin{abstract}
Objective. The aim of this study was to validate and compare the performance of serum paraquat level, severity index of paraquat poisoning (SIPP), Acute Physiology And Chronic Health Evaluation II (APACHE II), modified Simplified Acute Physiology Score II (MSAPS II), and modified Expanded Simplified Acute Physiology Score II (MSAPS IIe) calculated immediately after arrival on emergency department (ED) for assessing the mortality of acute paraquat poisoning. Methods. A retrospective study design was employed with the main outcome measure being mortality from year 2001 to 2010. MSAPS II and MSAPS IIe were employed in that assessment of the 24-hour urine output were not included. The performance of APACHE II, MSAPS II, MSAPS IIe, serum paraquat level and SIPP for prediction of mortality in acute paraquat poisoning were compared. Results. A total of 102 patients were enrolled in the study. The area under the ROC curve for APACHE II (0.800) was statistically lower than those for MSAPS II, MSAPS IIe, SIPP and serum paraquat (0.879, 0.893, 0.924,and 0.951, respectively). The Hosmer-Lemeshow goodness-of-fit test $\mathrm{C}$ statistic revealed that APACHE II, MSAPS II, MSAPS IIe and serum paraquat level showed good calibrations (chi-square 8.477 and $\mathrm{p}=0.388$, chi-square 4.614 and $\mathrm{p}=0.798$, chi-squared 5.301 and $\mathrm{p}=0.725$, chisquared 1.009 and $\mathrm{p}=0.985$ respectively), but poor calibration for SIPP (chi-square 21.293 and $\mathrm{p}=0.006$ ). Conclusion. Serum paraquat level is still the most reliable prognosis factor in acute paraquat poisoning. But MSAPS II or MSAPS IIe calculated immediately after arrival on ED may be helpful to predict mortality in acute paraquat poisoning especially when hospital has no facility to measure serum paraquat level.
\end{abstract}

Keywords Paraquat; Poisoning; Death

\section{Introduction}

Paraquat is a highly toxic herbicide that has been widely used in many countries since the 1960s. Many treatment modalities have been developed for paraquat poisoning, but the efficacy of such therapeutic methods is uncertain. Serum paraquat level and its relationship to time of ingestion is the most reliable prognosis factors in acute paraquat poisoning. ${ }^{1,2}$ Previous study showed that severity index of paraquat poisoning (SIPP) was a significant prognostic factor in acute paraquat poisoning. ${ }^{3}$ But many hospitals do not have the facilities to measure the serum paraquat levels, it's practical value is limited. Huang et al. ${ }^{4}$ showed that Acute Physiology And Chronic Health Evaluation II (APACHE II) ${ }^{5}$ calculated on 24 hours after admission was a good predictor of in-hospital mortality in patients with acute paraquat poisoning. But some patients died within 24 hours and this study only involved patients who survived more than 24 hours. Simplified Acute Physiology Score II (SAPS II) ${ }^{6}$ is another widely used severity scoring system as well as APACHE II

Received 28 March 2011; accepted 26 August 2011.

Address correspondence to Dr. Chi Keung Chan, Poison Information Center, Christian Hospital, 130 Hip Wo Street, Kwun Tong, Kowloon, Hong Kong. E-mail: chanck3@ @a.org.hk for patients admitted to intensive care units (ICU) to predict mortality. SAPS II requires a limited number of parameters and take less time to calculate. Expanded Simplified Acute Physiology Score II (SAPS IIe) was developed by adding six variables that were potentially associated with mortality to improve mortality prediction in ICU.7 Although APACHE II calculated on 24 hours after admission was known as good predictor of in-hospital mortality in patients with acute paraquat poisoning, the role of severity scoring systems such as APACHE II, SAPS II and SAPS IIe calculated immediately after arrival on ED in patients with acute paraquat poisoning is still unknown.

The aim of this study was to validate and compare the performance of APACHE II, modified SAPS II (MSAPS II), modified SAPS IIe (MSAPS IIe) calculated immediately after arrival on ED for assessing the mortality of acute paraquat poisoning.

\section{Methods}

\section{Study design and patients}

This study was approved by the medical ethics committee of our hospital. This study was conducted at a universityaffiliated, urban teaching hospital with 1,100 beds. According 


\section{Total 113 patients}

with acute paraquat poisoning during the 10 -year period

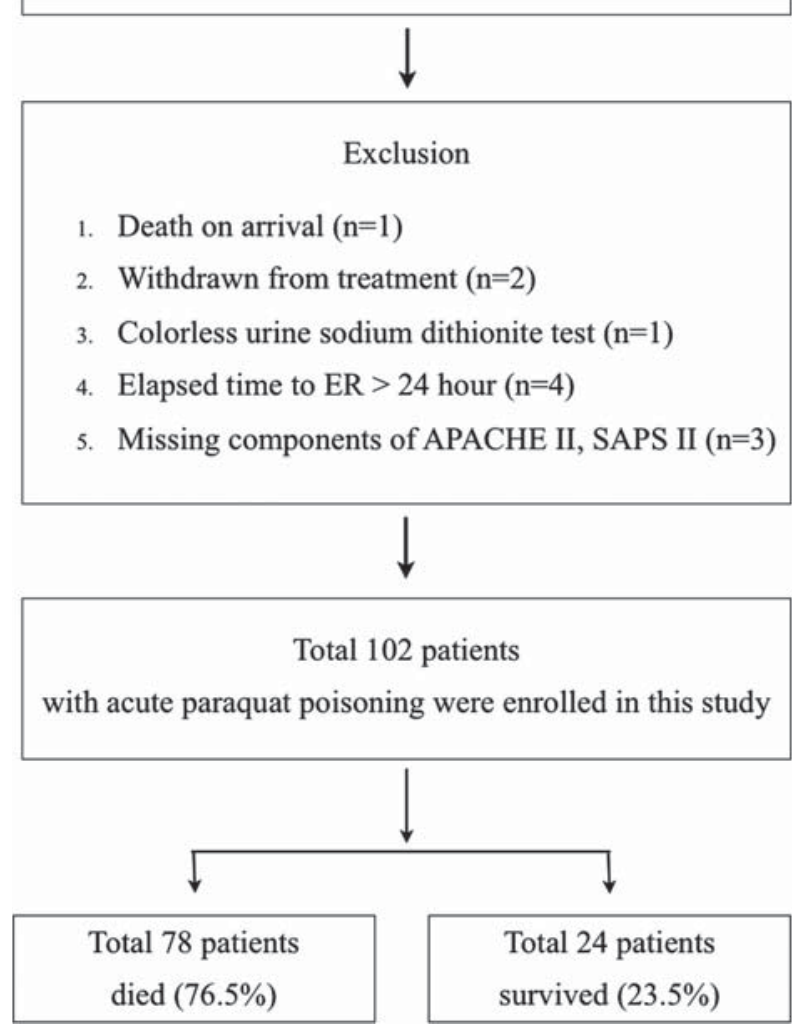

Fig. 1. A Flow chart of our study.

to the annual ED census, ED treats about 90,000 patients each year. A retrospective study design was employed with the main outcome measure being mortality. Because some patients with paraquat poisoning were discharged hopelessly and died within 7 days after discharge, mortality was defined as death occurring in the hospital or within 7 days after discharge. A concurrent study was conducted by collecting data on consecutive patients who visited ED after ingestion of paraquat over 10 year period (January 2001 to December 2010). The cases were collected from poisoning patient log book of our department, electronic patient log book in ED, laboratory alert, ICD (International Classification of Disease)-10 code. Paraquat poison- ings were confirmed by urine sampling using sodium dithionite reaction test and the serum paraquat levels were measured by high performance liquid chromatography (Acclaim ${ }^{\circledR}$, Bio-Rad Laboratories, USA) with a detection limit of $0.01 \mu \mathrm{g} / \mathrm{ml} .{ }^{8}$ The patients were managed with our standard treatment protocol which included gastric lavage, followed by infusion of $1 \mathrm{~g} / \mathrm{kg}$ activated charcoal, pulse therapies of cyclophosphamide $(1 \mathrm{~g} /$ day for 2days) and methylprednisolone (1 g/day for 3days), charcoal hemoperfusion. Charcoal hemoperfusion was given to those who had urine paraquat tests that showed strong dark or navy blue coloring except them who did not give informed consent for charcoal hemoperfusion $(n=28)$. Patients who were excluded from the present study included those who were dead on arrival $(n=1)$, who were withdrawn from treatment $(\mathrm{n}=2)$, who had colorless urine sodium dithionite test $(\mathrm{n}=1)$ and who arrived at the ED more than 24 hours after intoxication $(n=4)$ and who had missing components of APACHE II, SAPS II $(\mathrm{n}=3)$ (Fig. 1).

\section{Definition and Data Collection}

The definitions of MSAPS II and MSAPS IIe were that assessments of 24-hour urine output were excluded from SAPS II and SAPS IIe because it was unable to measure 24-hour urine output immediately after arrival on ED. APACHE II, MSAPS II, MSAPS IIe were calculated based on clinical and laboratory data which were collected immediately after arrival on ED. All the data and score were collected and calculated by one well-trained abstractor using electronic medical records. This process was performed repeatedly and rechecked for accuracy by the authors. Serum paraquat levels were also measured immediately after arrival on ED. The severity of paraquat poisoning was estimated quantitatively using the Severity Index of Paraquat Poisoning (SIPP), which was calculated by multiplying the elapsed time (hours) from ingestion to arrival by the serum paraquat level $(\mu \mathrm{g} / \mathrm{ml}){ }^{3}$

\section{Statistical analysis}

Data were presented as medians. For continuous variables, mean values were compared using Mann-Whitney U test. Pearson's chi-square statistic was used to test for the statistical significance of categorical variables. A p value less

Table 1. Baseline characteristics of patients $(n=102)$.

\begin{tabular}{|c|c|c|c|}
\hline & Survivors $(n=24)$ & Non-survivors $(\mathrm{n}=78)$ & $\mathrm{P}$ value \\
\hline Age (years) ${ }^{*}$ & $45(18,68)$ & $61(25,87)$ & $<0.01$ \\
\hline Gender (M:F) & & & 0.33 \\
\hline Male & $13(54.2 \%)$ & $52(66.7 \%)$ & \\
\hline Time from ingestion to ED (hour) ${ }^{*}$ & $2.70(0.5,19.2)$ & $2.00(0.3,19.0)$ & 0.18 \\
\hline Serum paraquat level $(\mu \mathrm{g} / \mathrm{ml})^{*}$ & $0.69(0.06,4.60)$ & $15.00(0.20,3010.00)$ & $<0.01$ \\
\hline SIPP $^{*}$ & $1.28(0.10,56.58)$ & $40.95(0.50,2107.00)$ & $<0.01$ \\
\hline MSAPS $\mathrm{II}^{*}$ & $1.99(1.23,2.81)$ & $3.25(1.24,6.20)$ & $<0.01$ \\
\hline Mechanical ventilation & $3(12.5 \%)$ & $15(19.2 \%)$ & 0.55 \\
\hline Hemoperfusion & $13(54.2 \%)$ & $45(57.7 \%)$ & 0.47 \\
\hline
\end{tabular}

*Data are presented as median (minimum, maximum). 
A
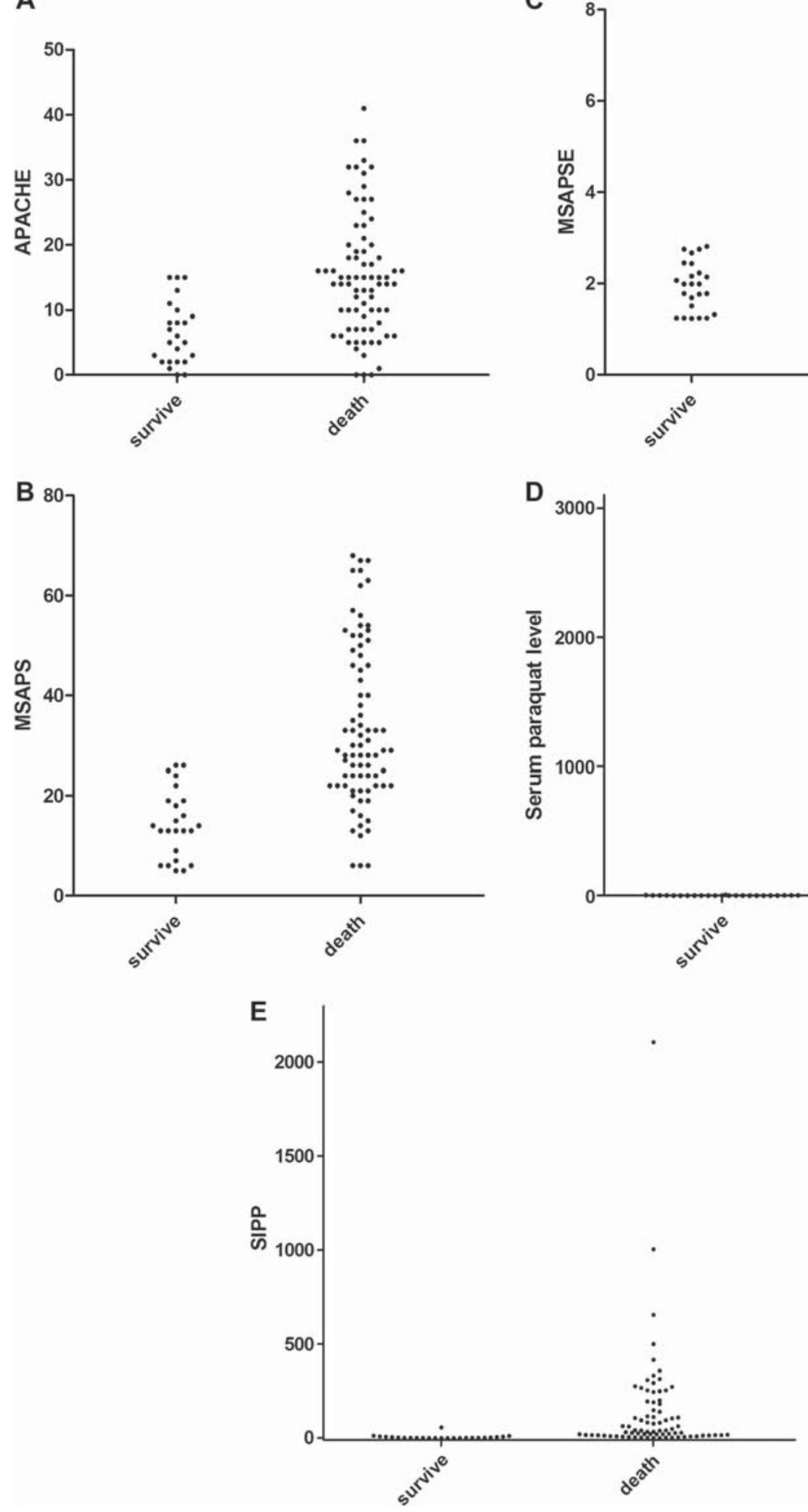

Fig. 2. Scatter plots of survivors vs. deaths.
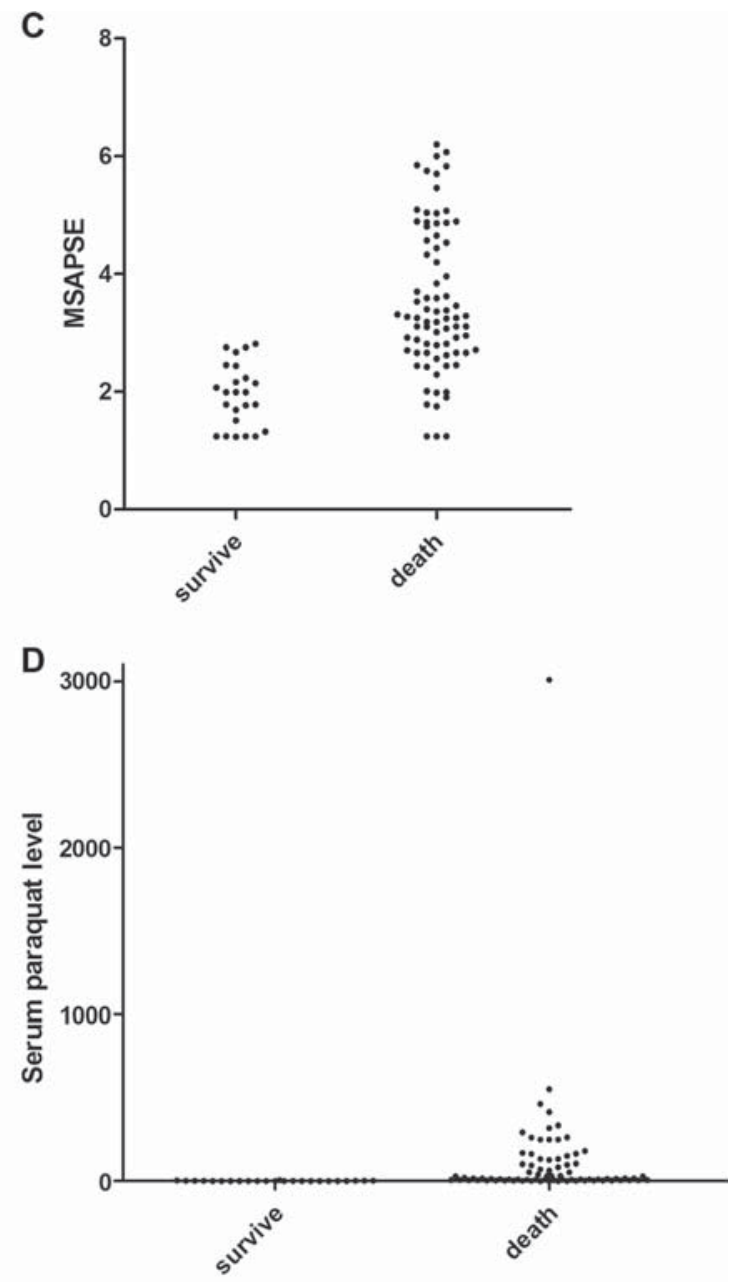

than 0.05 was considered statistically significant. The ability and accuracy of the models for mortality prediction were determined by examining their discrimination and calibration. A computation of the area under the receiver operating characteristics (ROC) curve for APACHE II, MSAPS II , MSAPS IIe, serum paraquat level and SIPP were performed and compared to test discrimination. The sensitivity, specificity, positive predictive value, negative predictive value for APACHE II, MSAPS II, MSAPS IIe, serum paraquat level and SIPP were determined. The best cut-off points for predicting mortality were identified as the score giving the best Youden index (sensitivity + specificity 1 ) for each models. The Youden index evaluates the diagnostic efficacy of a test and if the index is closer to 1 , its diagnostic value is higher. The Hosmer-Lemeshow goodness-of-fit $\mathrm{C}$ statistics was used to determine the calibration. A $p$ value greater than 


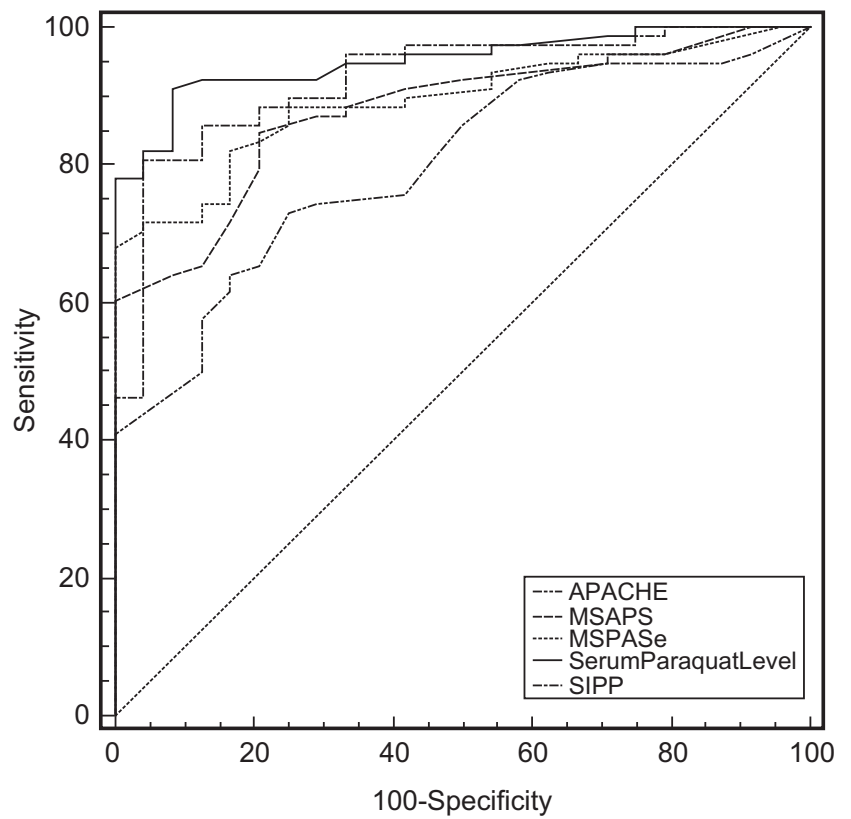

Fig. 3. ROC curves for APACHE II, MSAPS II, MSAPS IIe, SIPP and serum paraquat level on prediction ofmortality.

0.05 indicates a model with good calibration. All statistical analyses were performed using SPSS 12.0 (SPSS, Chicago, USA) and Medcalc 11.6 (Medcalc software, Mariakerke, Belgium).

\section{Results}

A total of 102 patients were enrolled in the study. Patients characteristics and univariate analysis for predicting mortality are shown in Table 1 . The comparison of serum paraquat level, SIPP, APACHE, MSAPS II and MSAPS IIe were illustrated with scatter plots of survivors vs deaths (Fig. 2). A total of 78 patients died and overall mortality was $76.5 \%$. The primary causes of deaths were circulatory failure $(n=60)$ and hypoxemia $(\mathrm{n}=18)$.

The overall discriminatory capabilities, as measured by the area under the ROC curve, were generally good for all models. The area under the ROC curve was 0.800 (SE 0.045, 95\% CI $=0.7100 .873$ ) for APACHE II, 0.879 (SE 0.034, $95 \% \mathrm{CI}=0.8000 .935$ ) for MSAPS II, 0.893 (SE 0.031, $95 \% \mathrm{CI}=0.8160 .946$ ) for MSAPS IIe, 0.951 (SE 0.020, $95 \% \mathrm{CI}=0.8900 .984$ ) for serum paraquat level and 0.924 (SE $0.026,95 \% \mathrm{CI}=0.8540 .967)$ for SIPP (Fig. 3, Table 2).
Although all the models had good discriminative power in the prediction of mortality, the area under the ROC curve for APACHE II was statistically lower than the area under the ROC curves for MSAPS II, MSAPS IIe, SIPP and serum paraquat level, respectively (Table 3). And although statistically insignificant, area under the ROC curve for MSAPS IIe was greater than that for MSAPS II. Table 2 also shows the sensitivity, specificity, positive predictive value, positive and negative predictive value and Youden index at each cut-off point.

To estimate the calibration power of each model, the Hosmer-Lemeshow goodness-of-fit test C statistic was used (Table 4). The Hosmer-Lemeshow goodness-of-fit test C statistic revealed that APACHE II, MSAPS II, MSAPS IIe and serum paraquat level showed good calibration powers (chisquare 8.477 and $p=0.388$, chi-square 4.614 and $p=0.798$, chi-squared 5.301 and $\mathrm{p}=0.725$, chi-squared 1.009 and $\mathrm{p}=0.985$, respectively). However, the Hosmer-Lemeshow goodness-of-fit $\mathrm{C}$ statistics showed poor calibration power for SIPP (chi-square 21.293 and $p=0.006$ ) and this finding indicated a significant lack of fit for SIPP.

\section{Discussion}

In present study, serum paraquat level was the most accurate prognostic factor to predict mortality after acute paraquat. This result is in concordance with results of other studies ${ }^{1,2}$ and serum paraquat level is still the most reliable prognosis factors in acute paraquat poisoning.

Serum paraquat level and its relationship to time data have been used to predict outcome from acute paraquat poisoning for nearly three decade since Proudfoot et al produced a normogram in $1979 .{ }^{1}$ Despite the fact that serum paraquat level and its relationship to time of ingestion is the most reliable prognosis factors in acute paraquat poisoning, there are some limitations using serum paraquat level and its relationship to time data to predict outcome. The first limitation is fact that estimation of the time interval since ingestion of paraquat is prone to error. Serum paraquat level will decline rapidly during first few hours after ingestion, a error in the estimation of time interval since ingestion of paraquat even if for few minutes may radically alter SIPP. But most important limitation using serum paraquat level to predict outcome is that its measurement is not readily available in most hospitals. For this reason, it's practical value is limited

Table 2. Discriminative power of each models for predicting mortality.

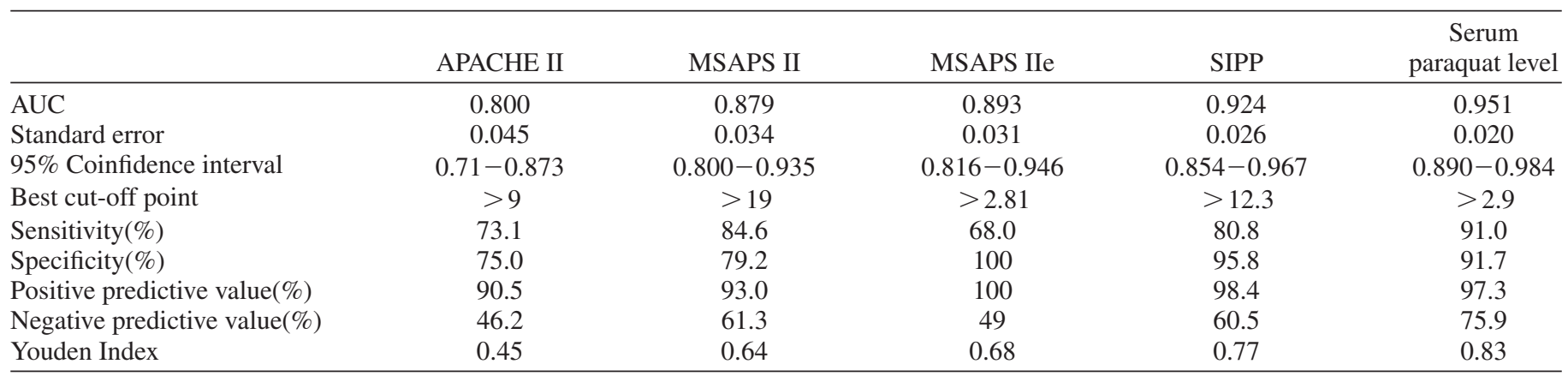


Table 3. Comparison of area under the ROC curve for each prognostic models ( $\mathrm{p}$ value*).

\begin{tabular}{lcccc}
\hline & $\begin{array}{c}\text { Serum } \\
\text { paraquat level }\end{array}$ & SIPP & MSAPS IIe & MSAPS II \\
\hline APACHE & 0.001 & 0.010 & 0.013 & 0.047 \\
MSAPS II & 0.048 & 0.240 & 0.224 & \\
MSAPS IIe & 0.097 & 0.399 & & \\
SIPP & 0.086 & & & \\
\hline
\end{tabular}

*P value by pair-wise comparison using Medcalc 11.6 (Medcalc software, Belgium).

and many studies were performed to predict prognosis of paraquat poisoning using usual data or tests such as blood biochemistry. Bismuth el al showed that the prognosis of the paraquat poisoning depended on renal function and acidbase balance. ${ }^{9}$ Age and white blood cell count at admission are also known as predictors of outcomes in acute paraquat poisoning. ${ }^{10}$ These kinds of predictors were included in variables of APACHE II and SAPS II. The most striking study regarding prediction of prognoses in acute paraquat poisoning is that by Huang et al. ${ }^{4}$ They successfully applied APACHE II which were calculated on 24 hours after admission in predicting the in-hospital mortality of paraquat poisoning, and they concluded that the APACHE II score was a simple, reproducible and practical tool for evaluating the severity of acute paraquat poisoning. In their study, the performance of each prognostic models were evaluated by their discrimination using the areas under the ROC curves. But they did not evaluate calibration of each prognostic models. On the other hand, we calculated APACHE II, MSAPS II and MSAPS IIe immediately after arrival on ED and used the Hosmer-Lemeshow goodness-of-fit test C statistic to determine the calibration. So there were at least 24 hours of time intervals of calculation between two studies. In our study, although all the models had good discriminative power in the prediction of mortality, the area under the ROC curve for APACHE II was statistically lower than those for MSAPS II, MSAPS IIe, SIPP and serum paraquat level. This means that the discrimination power of MSAPS II or MSAPS IIe calculated immediately after arrival on ED is superior to that of APACHE II calculated immediately after arrival on ED. Although statistically insignificant, the area under the ROC curve for MSAPS IIe was greater than that of MSAPS II. Additional six variables in SAPS $\mathrm{II}^{7}$ might influence this result. In addition to evaluation of discrimination power, we also evaluated the calibration of each prognostic models using the Hosmer-Lemeshow goodness-of-fit test $\mathrm{C}$ statistic. The Hosmer-Lemeshow goodness-of-fit test $\mathrm{C}$ statistic revealed that APACHE II, MSAPS II, MSAPS IIe and serum paraquat

Table 4. The Hosmer-Lemeshow goodness-of-fit test $\mathrm{C}$ statistic for each models

\begin{tabular}{lcc}
\hline & Chi square & P value \\
\hline APACHE II & 8.477 & 0.388 \\
MSAPS II & 4.614 & 0.798 \\
MSAPS IIe & 5.301 & 0.725 \\
Serum paraquat level & 1.099 & 0.985 \\
SIPP & 21.293 & 0.006 \\
\hline
\end{tabular}

level showed good calibration to predict mortality in acute paraquat poisoning, but SIPP showed poor calibration. This means that SIPP might be not a good model for prediction of mortality in acute paraquat poisoning although SIPP showed good discriminative power. But it is unclear why SIPP showed poor calibration. The reason may be as follows. First, relatively small sample size might affect this result. Second, because patients or family might not remember the exact time of ingestion, there might be some errors in the estimation of the time interval since ingestion of paraquat which was prone to error as described above. Lastly, SIPP model itself might have a poor calibration power to predict mortality in acute paraquat poisoning.

We reviewed some articles to see if the performance of APACHE II or SAPS II in acute paraquat poisoning was similar to that in other populations (Table 5). The area under the ROC curves for APACHE II ranged from 0.803 to 0.884 and those for SAPS II ranged from 0.772 to 0.857 in various populations. ${ }^{11-16}$ Although time of calculation of scores in other populations was different from that in our study, the performances of APACHE II and SAPS II in other populations were similar to those in acute paraquat poisoning. It seems that the best cut-off points for mortality in other populations were higher than those in acute paraquat poisoning (Table 5). In our study, predicted mortality calculated by APACHE II ranged from 5.8\% to $9.9 \%$ but observed mortality was $65.2 \%$ in the range of 5 to 9 point of APACHE II score. This results indicated that the predicted mortality calculated by APACHE II or SAPS II might underestimate mortality in acute paraquat poisoning compared to its use in other population. Although these severity scoring systems might be helpful to predict mortality in acute paraquat poisoning, the application of these severity scoring systems to acute paraquat poisoning need special consideration and we must not rely on the predicted mortality itself calculated by these scoring systems in acute paraquat poisoning.

The overall mortality in our study was $76.5 \%$ and this was higher than that in previous study. ${ }^{17}$ Because serum paraquat level is the most accurate prognostic factor in acute paraquat poisoning, this difference in mortality might be primarily caused by differences in severity of paraquat poisoning. For example, median and maximum serum paraquat level of enrolled patients in current study were $8.15 \mu \mathrm{g} / \mathrm{ml}, 3010.00$ $\mu \mathrm{g} / \mathrm{ml}$, respectively. These paraquat levels were higher than those in previous study(median $1.9 \mathrm{mg} / \mathrm{L}$, maximum $25.7 \mathrm{mg} / \mathrm{L}) .{ }^{17}$

In current study, not all the patients received pulse therapy of steroid and cyclophosphamide completely because a total of 19 patients died within 72 hours after admission. The cause of death in these 19 patients was circulatory failure. These 19 patients might have died regardless of pulse therapy with steroid and cyclophosphamide because their serum paraquat levels were very high (median $250 \mu \mathrm{g} / \mathrm{ml}$, minimum $102 \mu \mathrm{g} /$ $\mathrm{ml}$, maximum $3010 \mu \mathrm{g} / \mathrm{ml}$ ).

According to our results, MSAP II or MSAPS IIe calculated immediately after arrival on ED were good models for prediction of mortality in acute paraquat poisoning. Because all the parameter of MSAPS II and MSAPS IIe could be 
Table 5. Performance of APACHE II \& SAPS II in various populations.

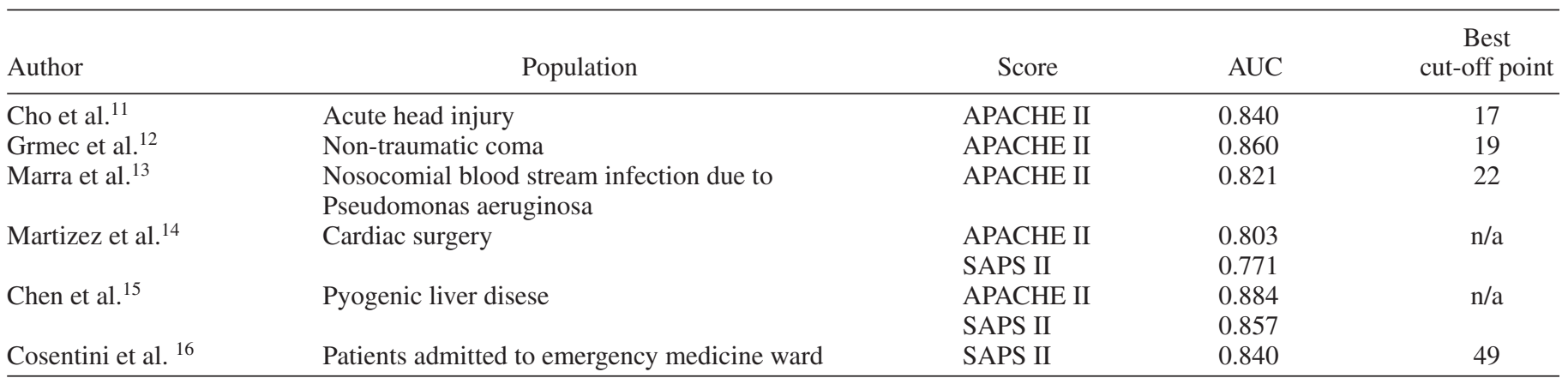

easily acquired in most ED setting, MSAPS II or MSAPS IIe calculated immediately after arrival on ED can be helpful to predict mortality in acute paraquat poisoning, especially when the hospital has no facility to measure serum paraquat level. And earlier prediction could be possibly compared to the calculation of APACHE II on 24 hours of admission. ${ }^{4}$

Our study has some limitations. We gathered 102 cases of acute paraquat poisoning over 10 years. While this is a high number for paraquat poisoning, it is rather a low one for the statistical analysis. Although meaningful statistical analysis could be performed, our study was probably not large enough to come to firm conclusions - nevertheless it is an inherent limitation in almost all toxicology research. Another limitation was that our study was a retrospective in its design, so we could not control the bias, and this might have affected the results. Further large studies are needed to overcome these limitations.

In spite of positive results with MSAPS II and MSAPS IIe, serum paraquat level is still the most reliable prognosis factor in acute paraquat poisoning, and these kinds of severity scoring system cannot replace serum paraquat level and must be considered as references.

\section{Conclusions}

In present study, serum paraquat level is the most accurate prognostic factor to predict mortality after acute paraquat. But MSAPS II or MSAPS IIe calculated immediately after arrival on ED had good discrimination and calibration powers in the prediction of mortality from acute paraquat poisoning. So MSAPS II or MSAPS IIe calculated immediately after arrival on ED may be helpful to predict mortality in acute paraquat poisoning especially when hospital has no facility to measure serum paraquat level. Further studies are needed to confirm these results.

\section{Declaration of interest}

The authors report no conflicts of interest. The authors alone are responsible for the content and writing of this paper.

\section{References}

1. Proudfoot AT, Stewart MS, Levitt T, Widdop B. Paraquat poisoning: significance of plasma-paraquat concentration. Lancet 1979; 2:330-332.
2. Jones AL, Elton R, Flanagan R. Multiple logistic regression analysis of plasma paraquat concentration as a predictor of outcome in 375 cases of paraquat poisoning. QJM 1999; 92:573-578.

3. Sawada Y, Yamamoto I, Hieokane T, Nagai Y, Satoh Y, Ueyama M. Severity index of paraquat poisoning. Lancet 1988; 1:1333.

4. Huang NC, Hung YM, Lin SL, Wann SR, Hsu CW, Ger LP, et al. Further evidence of the usefulness of Acute Physiology and Chronic Health Evaluation II scoring system in acute paraquat poisoning. Clin Toxicol 2006; 44(2):99-102.

5. Knaus WA, Draper EA, Wagner DP, Zimmerman JE. APACHE II: a severity of disease classification system. Critical Care Medicine 1985; 13(10):818-829.

6. Le Gall JR, Lemeshow S, Saulnier F. A New Simplified Acute Physiology Score (SAPS II) Based on a European/North American Multicenter Study. JAMA 1993; 270(24):2957-2963.

7. Le Gall JR, Neumann A, Hemery F, Bleriot JP, Fulgencio JP, Garrigues B, et al. Mortality prediction using SAPS II: an update for French intensive care units. Critical Care 2005; 9(6):R645-R652.

8. Croes K, Martens F, Desmet K. Quantitation of paraquat in serum by HPLC. J Anal Toxicol 1993; 17(5):310-312.

9. Bismuth C, Garnier R, Dally S, Fournier PE, Scherrmann JM. Prognosis and treatment of paraquat poisoning: a review of 28 cases. J Toxicol Clin Toxicol 1982; 19:461-474.

10. Kaojarern S, Ongphiphadhanakul B. Predicting outcomes in paraquat poisonings. Vet Hum Toxicol 1991; 33:115-118.

11. Cho DY, Wang YC. Comparison of the APACHE III, APACHE II and Glasgow Coma Scale in acute head injury for prediction of mortality and functional outcome. Intensive Care Med 1997; 23(1): 77-84.

12. Grmec S, Gasparovic V. Comparison of APACHE II, MEES and Glasgow Coma Scale in patients with nontraumatic coma for prediction of mortality. Acute Physiology and Chronic Health Evaluation. Mainz Emergency Evaluation System. Crit Care 2001; 5(1): $19-23$.

13. Marra AR, Bearman GM, Wenzel RP, Edmond MB. Comparison of severity of illness scoring systems for patients with nosocomial bloodstream infection due to Pseudomonas aeruginosa. BMC Infect Dis 2006; 6:132.

14. Martínez-Alario J, Tuesta ID, Plasencia E, Santana M, Mora ML. Mortality prediction in cardiac surgery patients: comparative performance of Parsonnet and general severity systems. Circulation 1999; 99(18):2378-2382.

15. Chen SC, Huang CC, Tsai SJ, Yen CH, Lin DB, Wang PH, et al. Severity of disease as main predictor for mortality in patients with pyogenic liver abscess. Am J Surg 2009; 198(2):164-172.

16. Cosentini R, Folli C, Cazzaniga M, Aliberti S, Piffer F, Grazioli L, et al. Usefulness of simplified acute physiology score II in predicting mortality in patients admitted to an emergency medicine ward. Intern Emerg Med 2009; 4(3):241-247.

17. Lin JL, Lin-Tan DT, Chen KH, Huang WH, Hsu CW, Hsu HH, Yen $\mathrm{TH}$. Improved survival in severe paraquat poisoning with repeated pulse therapy of cyclophosphamide and steroids. Intensive Care Med. 2011; 37(6):1006-1013. 